can learn from labelled datasets, and then provide independent interpretations on new cases, but often without traceability to how such conclusions were made. This 'black box' behaviour is not desirable for clinical applications. We propose a novel framework for visualising and guiding the AI attention, using artefact detection in cardiac T1-mapping as an example - a critical quality assurance step for clinically-robust T1 determinations.

Method We utilised an AI attention visualisation framework. This serves as an 'eye tracker' and reveals where the neural network 'looks' when scoring artefacts. The technique adds an essential accountability aspect to the $\mathrm{CNN}$ by producing additional evidence to validate the decision-making process. Beyond simply observing the AI attention maps, we provided additional direct guidance on the attention of the CNN, instructing the machine where to look, similar to training a human operator. Results We demonstrate an application in automated T1-mapping artefact detection of the 6 AHA segments in mid-ventricular slices (figure 1a). The AI 'eye tracker' detected an illtrained CNN not paying attention to myocardium (figure $1 \mathrm{~b}$ ). A well-trained CNN learned from the training data to pay attention to the 6 myocardial segments, but with distraction by other image features (red arrows, figure 1c) and inaccuracy (yellow arrows). The proposed solution is a CNN trained with additional guidance to pay attention to the correct structures and avoid distractions (figure 1d).

Conclusion CNN designed with both visualisation in perception and guidance on attention to relevant anatomical structures can lead to significantly more transparent and accountable AI, therefore more reliable for clinical practice.

\section{MANGANESE ENHANCED T1 MAPPING OF CALCIUM HOMEOSTASIS IN ACUTE MYOCARDIAL INFARCTION}

${ }^{1,2} \mathrm{NH}$ Jasmin, ${ }^{1} \mathrm{M}$ Zaw-Thin, ${ }^{1} \mathrm{MF}$ Lythgoe, ${ }^{3} \mathrm{~S}$ Davidson, ${ }^{1} \mathrm{DJ}$ Stuckey. ${ }^{1}$ University College London, Centre for Advanced Biomedical Imaging, London, UK; ${ }^{2}$ Universiti Sultan Zainal Abidin, School of Medical Imaging, Terengganu Darul Iman, Malaysia; ${ }^{3}$ University College London, Hatter Institute, London, UK

10.1136/heartjnl-2019-BSCMR.4
Introduction Manganese is a calcium analogue and potent T1contrast agent for MRI that enters cardiomyocytes through active calcium channels, thus reducing T1 in viable myocardium. ${ }^{1}$ We hypothesized that T1-mapping-MRI could measure $\mathrm{Mn}$ levels in the myocardium acutely after myocardial infarction (MI) allowing quantification of intracellular $\mathrm{Ca}$ response to ischemic injury.

Methods Mice received intraperitoneal injections of $0.10 \mathrm{mmol} / \mathrm{kg} \mathrm{MnCl}_{2} 40 \mathrm{~min}$ before MI. T1-mapping-MRI was performed at 1, 2 and 3 hours and 2 days after MI. R1 values $(1 / \mathrm{T} 1=$ the relaxivity of the tissue) were analysed from the area-at-risk of infarction segments (AAR-MI, $n=12$ ) and viable segments (Viable-MI, $\mathrm{n}=12$ ) of infarcted hearts, and naïve control heart (Viable-Naïve, $\mathrm{n}=12$ ). Imaging was performed using a 9.4T Agilent MRI system and a multi inversion time Look-Locker sequence in the short-axis orientation (TE/

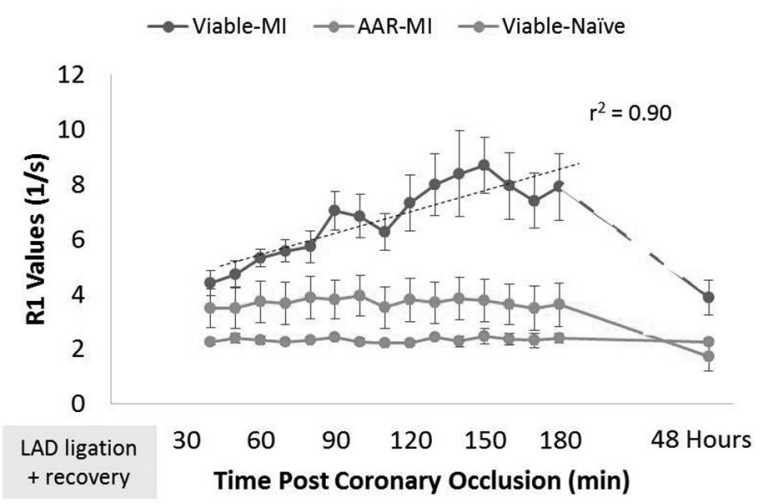

Abstract 4 Figure 2 R1 values in acute myocardial infarction after Mn infusion

R1 values in MI mouse hearts at 1, 2, 3 \& $48 \mathrm{~h}$ after myocardial infarction and in naïve mouse hearts at the same time points after $\mathrm{Mn}$ infusion. Viable-MI = Remote myocardium at 1 to 3 hours after occlusion, AAR-MI = Area at risk at 1 to 3 hours after occlusion, Viable-Naïve $=$ Remote myocardium in naïve control group

\section{(a) 1 Hour Post MI}

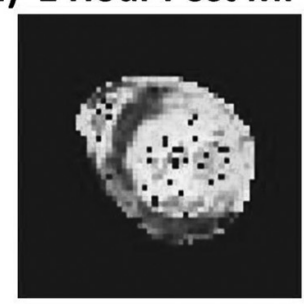

(b)

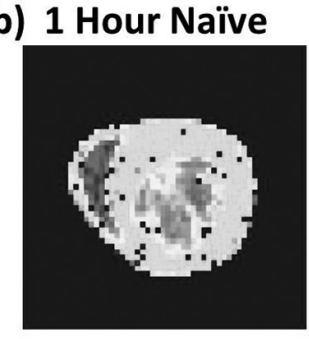

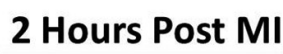
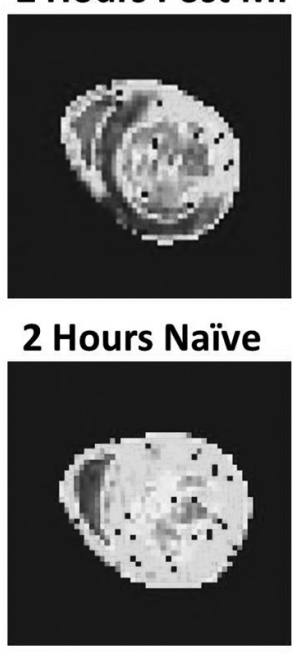

\section{Hours Post MI}
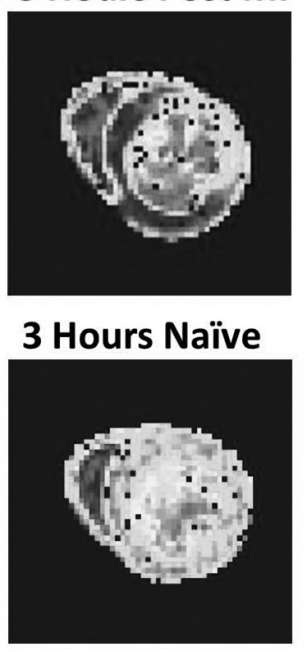
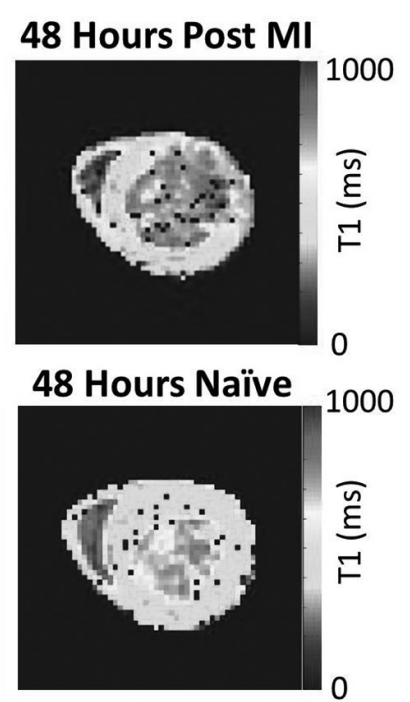

Abstract 4 Figure 1 T1 Maps of Mn uptake in acute myocardial infarction

T1 Maps acquired at 1,2,3 and $28 \mathrm{~h}$ after coronary occlusion (top) or in naïve mouse hearts (bottom). In the first hours after Ml, T1 in viable segments of infracted hearts was decreased compared with the area at risk of Ml and the naive myocardium owing to increased Mn uptake. By $48 \mathrm{~h}$, $\mathrm{T} 1$ in the infarct was higher than viable segments owing to reduced $\mathrm{Mn}$ uptake in the non-contractile ischemic zone 
$\mathrm{TR}=3.04 / 1.11 \mathrm{~ms}, 18$ inversion times at consecutive $\mathrm{R}$ waves, $10^{0}$ excitation pulse, slice thickness $=1.0 \mathrm{~mm}, \mathrm{FOV}=25.6 \times$ $25.6 \mathrm{~mm}$, matrix size $=128 \times 128)$ as described ${ }^{2}$

Results As soon as 1 hour after MI, R1 values increased in Viable-MI tissue compared with AAR-MI $(p<0.0001)$ and naïve controls. $\mathrm{R} 1$ values continued to rise in the Viable-MI at 2 and 3 hours $(p=0.02, p=0.01$. Figure 1 and 2$)$. When the same animals were imaged 2 days post-MI, R1 values were still significantly higher in the Viable-MI tissue compared with AAR-MI tissue $(\mathrm{p}=0.03)$. However, Viable-MI tissue had similar R1 to naïve hearts, while R1 in the infarcted AAR-MI was lower than of the naïve myocardium $(p=0.03$. Figure 1 and 2).

Discussion Acutely after ischemic injury a large increase in R1 (reflecting increased $\mathrm{Mn}^{2+}$ uptake) occurred in Viable-MI myocytes, likely due to elevated catecholamine levels acutely post-MI; increased cardiac work and thus increased $\mathrm{Ca}^{2+} / \mathrm{Mn}^{2}$

${ }^{+}$uptake. By 2 days the catecholamine storm has passed and $\mathrm{R} 1$ levels in the surviving myocardium normalise, while $\mathrm{Mn}$ uptake in the dead infarct region was reduced due to lack of functional myocytes.

Conclusions T1-Mapping Manganese-enhanced-MRI offers a valuable in vivo tool for optimisation of the many emerging pharmacological and biological interventions which aim to modulate $\mathrm{Ca}^{2+}$ homeostasis acuity after MI.

\section{REFERENCES}

1. Waghorn B, Edwards T, Yang Y, Chuang K-H, Yanasak N, Hu TC-C. Monitoring dynamic alterations in calcium homeostasis by T1-weighted and T1-mapping cardiac manganese-enhanced MRI in a murine myocardial infarction model. NMR in Biomedicine 2008;1102-1111.

2. Stuckey DJ, McSweeney SJ, Thin MZ, Habib J, Price AN, Fiedler LR, ... Schneider MD. T1 mapping detects pharmacological retardation of diffuse cardiac fibrosis in mouse pressure-overload hypertrophy. Circulation: Cardiovascular Imaging $2014 ; 7: 240-249$

\section{MANGANESE ENHANCED MRI CAN QUANTIFY MYOCARDIAL INFARCT SIZE EARLIER THAN GADOLINIUM ENHANCED MRI}

\begin{abstract}
${ }^{1,2} \mathrm{NH}$ Jasmin, ${ }^{1} \mathrm{M}$ Zaw-Thin, ${ }^{1} \mathrm{MF}$ Lythgoe, ${ }^{3} \mathrm{~S}$ Davidson, ${ }^{1} \mathrm{DJ}$ Stuckey. 'University College London, Centre for Advanced Biomedical Imaging, London, UK; ${ }^{2}$ Universiti Sultan Zainal Abidin, School of Medical Imaging, Terengganu Darul Iman, Malaysia; ${ }^{3}$ University College London, Hatter Institute, London, UK
\end{abstract}

\subsection{6/heartjnl-2019-BSCMR.5}

Introduction Late gadolinium enhanced MRI (LGE-MRI) can quantify infarct size after myocardial infarction (MI) but is non-specific and reflects the increased membrane rupture and extracellular space that develop post MI. ${ }^{1} \mathrm{Mn}$ is a potent T1contrast agent that enters myocytes through active calcium channels, thus reducing T1 in viable myocardium. ${ }^{2}$ This active process rapidly ceases under ischemia. Hence, we hypothesised that Mn-enhanced MRI (MEMRI) could quantify final infarct size earlier than LGE-MRI.

Methods Myocardial infarction was induced in 7 mice which then underwent MEMRI $\left(\mathrm{n}=4,0.1 \mathrm{mmol} / \mathrm{kg} \mathrm{MnCl}_{2}\right.$ ) or LGEMRI ( $\mathrm{n}=3,0.5 \mathrm{mmol} / \mathrm{kg}$ Gd-DTPA) at 1 hour post MI. All animals then underwent both MEMRI and LGEMRI at $\sim 24$ hours post MI with a contrast washout period of at least 5 hours between scans. Imaging was performed using a 9.4T Agilent MRI system and a multi-slice inversion recovery sequence in the short-axis orientation covering the whole left ventricle $\mathrm{TE} / \mathrm{TR}=3.04 / 1.11 \mathrm{~ms}, \mathrm{TI}=\sim 600 \mathrm{~ms}$ for MEMRI
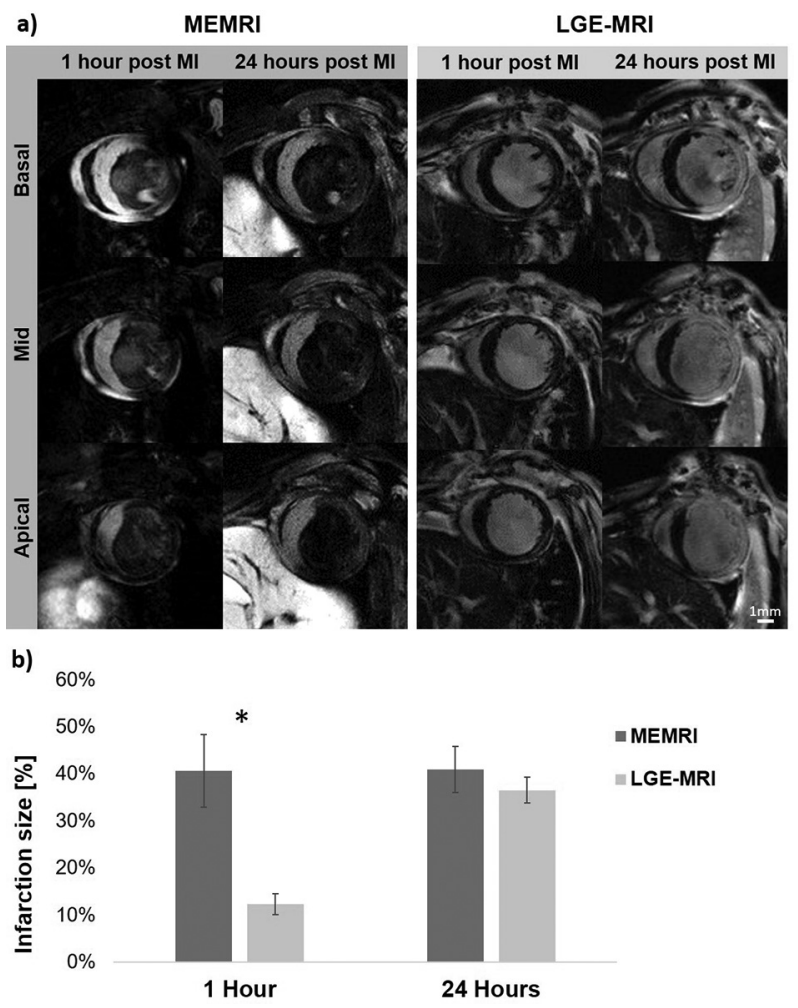

Abstract 5 Figure 1 MEMRI and LGE-MRI acquired at 1 and 24 hours post MI

At $1 \mathrm{~h}$ post $\mathrm{Ml}, \mathrm{Mn}$ uptake in viable myocardium allowed the estimated final infarct area to be distinguished, whilst only subtle enhancement was seen in LGE-MRI resulting in a smaller measure of occlusion zone. At $24 \mathrm{~h}$ post $\mathrm{Ml}$ the infarct shows hypoenhancement in MEMRI and hyperenhancement in LGE of similar sizes

\section{Day 1 post MI}

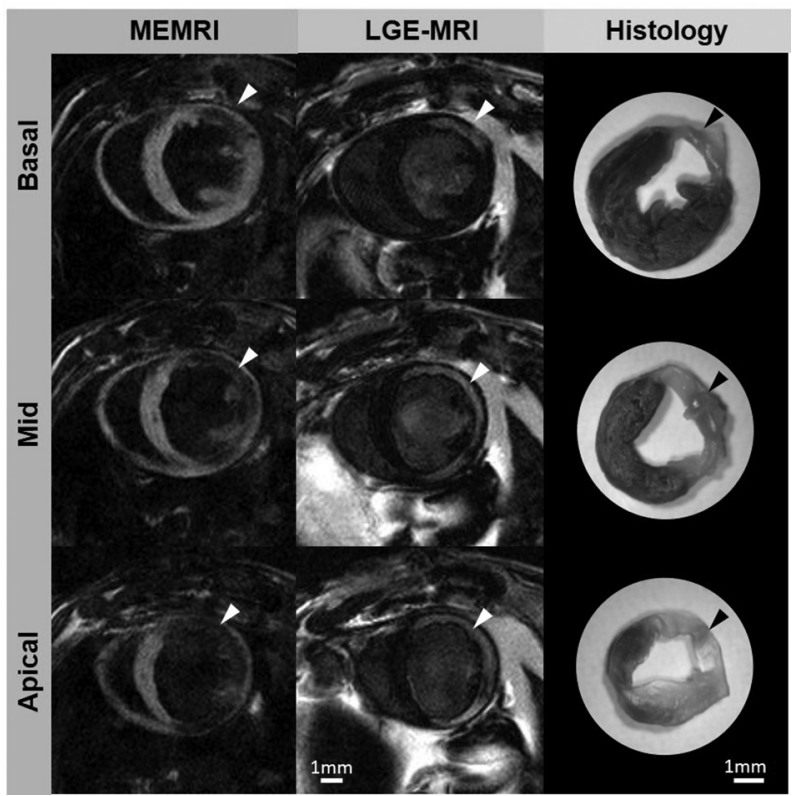

Abstract 5 Figure 2 Direct comparison of MEMRI and LGE-MRI Direct comparison of MEMRI and LGE-MRI acquired in the same animal at 22 and 27 hours after $\mathrm{Ml}$, respectively. Arrowhead shows hypoenhancement of the infarct in the MEMRI image and hyperenhancement of the infarct in LGE. In vivo data corresponded with TTC histological staining for infarct 